\title{
Whole blood RNA expression profiles in ovarian cancer patients with or without residual tumors after primary cytoreductive surgery
}

\author{
HELENA S. ISAKSSON ${ }^{1,3}$, BENGT SORBE $^{2}$ and TORBJÖRN K. NILSSON ${ }^{1,3}$ \\ Departments of ${ }^{1}$ Laboratory Medicine, Section for Clinical Chemistry, ${ }^{2}$ Gynecological Oncology, \\ Örebro University Hospital, and ${ }^{3}$ Health and Medical Sciences, Örebro University, SE-701 85 Örebro, Sweden
}

Received October 24, 2011; Accepted December 2, 2011

DOI: $10.3892 /$ or.2012.1680

\begin{abstract}
Significant improvements in the treatment results of ovarian cancer have been achieved during the last decades, but further improvements require additional methods identifying signs of the disease and its biological behavior, preferably by a simple blood test. We hypothesized that peripheral blood leukocytes may express genes that carry such clinical information. Therefore, we studied the relative gene expressions of 168 cancer- and metastasis-specific genes in blood samples from ovarian cancer patients with different prognoses after primary cytoreductive surgery. Total RNA was extracted from whole blood and the relative gene expression profile of 168 genes were analyzed using real-time qPCR assays. Two groups of patients were analyzed; one group with residual tumor mass after primary surgery, and one group where the tumor was macroscopically radically resected, resulting in no visible tumor mass left behind. The group with the remaining tumor mass after surgery showed significantly different gene expression profiles compared to the group with no remaining tumor mass. Differences were noted for the metastasis associated 1 family, member 2 gene (MTA2), the TNF, $\alpha$-catenin, interleukin $1 \beta$, the KiSS-1 metastasis suppressor and the matrix metalloproteinase 10 genes. All genes were downregulated with a fold-change between 1.15 to 1.57 ; there were no upregulated genes. Thus, a signature of genes involved in metastasis, invasion and inflammation was found to be significantly downregulated in native unstimulated blood leukocytes from ovarian cancer patients with a poor prognosis. Preoperatively it may serve as a guide to the biology of the tumor and postoperatively in the optimization of adjuvant treatment of ovarian cancer patients.
\end{abstract}

Correspondence to: Ms. Helena Isaksson, Department of Laboratory Medicine, Section for Clinical Chemistry, Örebro University Hospital, SE-701 85 Örebro, Sweden

E-mail: helena.isaksson@orebroll.se

Key words: seropapillary ovarian cancer, residual tumor, leukocyte gene expression, whole blood RNA expression

\section{Introduction}

Ovarian cancer is an important disease among gynecological malignancies. Despite a slowly decreasing incidence in many Western countries the prognosis is still unfavorable $(1,2)$, and the overall 5-year survival rate is approximately $50 \%$ at the best centers after primary cytoreductive surgery and combination chemotherapy with paclitaxel and carboplatin. Significant improvements in treatment results have been achieved during the last decades and further improvements are expected in the future for this disease. Many clinical trials are run to improve chemotherapy but also to incorporate new targeted therapy agents $(1,3,4)$.

Predictive and prognostic factors are important in guidance of expected survival but also for the choice of optimal primary treatment $(2,5)$. Some clinical prognostic factors are known, e.g. stage, histology, grade, and residual tumor after primary surgery. The volume of the residual tumor is one of the strongest prognostic factors for survival. The goal of the primary cytoreductive surgery is to reduce the tumor volume as much as possible to macroscopically no residual tumor or at least less than $1 \mathrm{~cm}$ tumor diameter of the remaining nodules. Centralized surgery and experienced tumor surgeons are important to achieve this goal, but the biology of the individual tumor is also thought to be of importance for the outcome of the surgery.

The biology of individual ovarian tumors can be characterized by their genetic profiles with up- and downregulation of important oncogenes and tumor suppressor genes. DNA and expression of RNA can be studied with microarray analyses of tissue samples from the tumor. Fresh or fresh-frozen tissue is generally needed for these analyses, but often these types of specimens are not available in routine clinical work. A much simpler way would be to analyze whole blood samples from the individual patient. There is a growing experience of messenger ribonucleic acid (mRNA) analyses of whole blood. Both blood leukocytes and circulating tumor cells may be the sources of mRNA in these analyses (6-8), but on a molar basis the leukocytes should regularly be the dominating source of mRNA. The mRNA from the leukocytes is thought to reflect more general and systemic reactions and tumor tissue mRNA specific tumor characteristics. Little is known about the extent 
Table I. Group age, tumor stage and residual tumor mass characteristics after primary surgery.

$$
\text { Group } 1(n=11) \quad \text { Group } 2(n=11)
$$

Age (mean, range)

Tumor stage, FIGO (n)

Remaining tumor mass after surgery
$60.7(43-85)$

IIIC (5), IV (5)

$>2 \mathrm{~cm}$
$57.4(40-78)$

IA (1), IB (1), IIB (1), IIC (4), IIIC (3)

None to which tumor characteristic expression patterns may actually be mirrored by the blood leukocytes. In the present study we tested the hypothesis that patients with a large remaining tumor mass after primary cytoreductive surgery and with a more advanced tumor stage express a different mRNA profile in their peripheral blood leukocytes before initiation of chemotherapy compared with patients successfully tumor-reduced to no visible tumor or with a less advanced tumor stage. An array of genes known to be potentially involved in tumorigenesis, and a metastasis array were used in the analyses. Detectable differences would reflect clinically important differences in tumor biology with regard to the grade of malignancy, invasiveness, angiogenesis, metastatic potential, and interaction with the host and could therefore be of potential aid in tailoring treatment and follow-up for the individual patient.

\section{Materials and methods}

Subjects. Blood samples were consecutively collected from all women with ovarian cancer (FIGO stage I-IV) admitted for treatment at the Department of Gynecological Oncology, University Hospital in Örebro, Sweden. All subjects had given their informed consent to take part in this study. The research project was approved by the Ethics Committee of UppsalaÖrebro, Sweden (\$198, 436/03, 2003-12-08). Twenty-two patients with seropapillary ovarian cancer were included in this study. The patients were divided into two groups (11 in each group), one with remaining tumor mass after surgery and at the start of chemotherapy (Group 1), and the other with all tumor mass removed by surgery (Group 2). The characteristics of the patients and tumors are presented in Table I.

Blood collection and extraction. The blood was collected in PAXgene tubes and the total RNA (ribonucleic acid) was extracted with the PAXgene Blood RNA kit (Qiagen Inc., Valencia, CA) in compliance with the manufacturer's instructions. Total RNA was assessed spectrophotometrically on an ND-1000 instrument (NanoDrop Technologies, Wilmington, DE, USA), quality controlled with the $260 / 280 \mathrm{~nm}$ quota and quantity measured as $n g / \mu 1$. We typically obtained $260 / 280$ ratios of 1.85-2.20 (mean 2.06 $\pm 0.21, \mathrm{n}=22$ ).

cDNA synthesis. One microgram of total RNA was transcribed into cDNA (single stranded complementary deoxyribonucleic acid) in a $20-\mu 1$ reaction volume using Omniscript reverse transcriptase for first-strand cDNA synthesis, two tube RT-PCR (reverse transcription polymerase chain reaction) (Omniscript ${ }^{\circledR}$ RT Kit for reverse transcription; Qiagen $\mathrm{GmbH}$, Hilden, Germany) using random hexamer primers and RNaseOUT Ribonuclease Inhibitor (Invitrogen, Karlsruhe,
Germany). The mastermix was prepared according to the manufacturer's instructions and incubated for $60 \mathrm{~min}$ at $37^{\circ} \mathrm{C}$ in a GeneAmp ${ }^{\circledR}$ PCR System 2700 PCR instrument (Applied Biosystems, Foster City, CA, USA).

PCR arrays. The selected PCR arrays include representative genes from the following biological processes involved in tumor genesis: cell cycle control and DNA damage repair, apoptosis and cell senescence, signal transduction molecules and transcription factors, angiogenesis, cell adhesion genes, extracellular matrix proteins, cell growth and proliferation genes, transcription factors and regulators, invasion and metastasis. The PCR array is based on a regular 96-well PCR plate, each well containing a different assay for one of the genes of interest. The RT ${ }^{2}$ SABiosciences Profiler PCR Array, Human Cancer PathwayFinder (SABioscience, Frederick, MD, USA, cat. no. PAHS-033) and the Human Tumor Metastasis (SABioscience, cat. no. PAHS-028) each profiles the expression of 84 genes representative of the biological processes listed above. The remaining 12 wells on each array contain housekeeping genes, controlling for contamination and RT-PCR efficiency. Both arrays are used together with the $\mathrm{RT}^{2}$ Real-Time SYBR Green/ ROX, PCR master mix (SABiosciences, cat. no. PA-012). All reactions were carried out according to the manufacturer's instructions on a Real-Time PCR instrument, ABI 7500 Fast (Applied Biosystems, Weiterstadt, Germany). As housekeeping genes we used RPL13A, GAPDH for the Human Cancer PathwayFinder Array and for the Human Tumor Metastasis array we used HPRT1, RPL13A, GAPDH and ACTB.

$\Delta \Delta C_{t}$ analysis method, fold-change. All threshold cycle values $\left(\mathrm{C}_{t}\right)$ reported as $>35$ or as not detected were considered as a negative call, according to the manufacturer's recommendation. The inverse proportional relationship between the $C_{t}$ and the original gene expression level (L), and the doubling of the amount of product with every PCR cycle, can be expressed as $\mathrm{L}=2^{-\mathrm{Ct}}$ for each gene of interest. To further normalize the expression level of a gene of interest (GOI) to a housekeeping gene $(\mathrm{HKG})$ the following calculations were carried out:

$$
\frac{2^{-\mathrm{Ct}(\mathrm{GOI})}}{2^{-\mathrm{Ct}(\mathrm{HKG})}}=2^{-[\mathrm{Cl}(\mathrm{GOI})-\mathrm{Ct}(\mathrm{HKG})]}=2^{-\Delta \mathrm{Ct}}
$$

To determine the fold-change in gene expression, the normalized expression of the GOI in the experimental sample (expt) was divided by the normalized expression of the same GOI in the control sample (control). The formula used was as follows:

$$
\frac{2^{-\mathrm{C}(\mathrm{GO}) \text { expt }} / 2^{-\mathrm{Cl}(\mathrm{HKG}) \operatorname{expt}}}{2^{-\mathrm{Cl}(\mathrm{GOI}) \text { control }} / 2^{-\mathrm{Cl}(\mathrm{HKG}) \text { control }}}=\frac{2^{-[\mathrm{C}(\mathrm{GOI})-(\mathrm{HKG})] \operatorname{expt}}}{2^{-[\mathrm{C}(\mathrm{GOI})-\mathrm{Cl}(\mathrm{HKG})] \text { control }}}=\frac{2^{-\Delta \mathrm{Cl} \text { expt }}}{2^{-\Delta \mathrm{Cl} \text { control }}}=2^{-\Delta \Delta \mathrm{Cl}}
$$



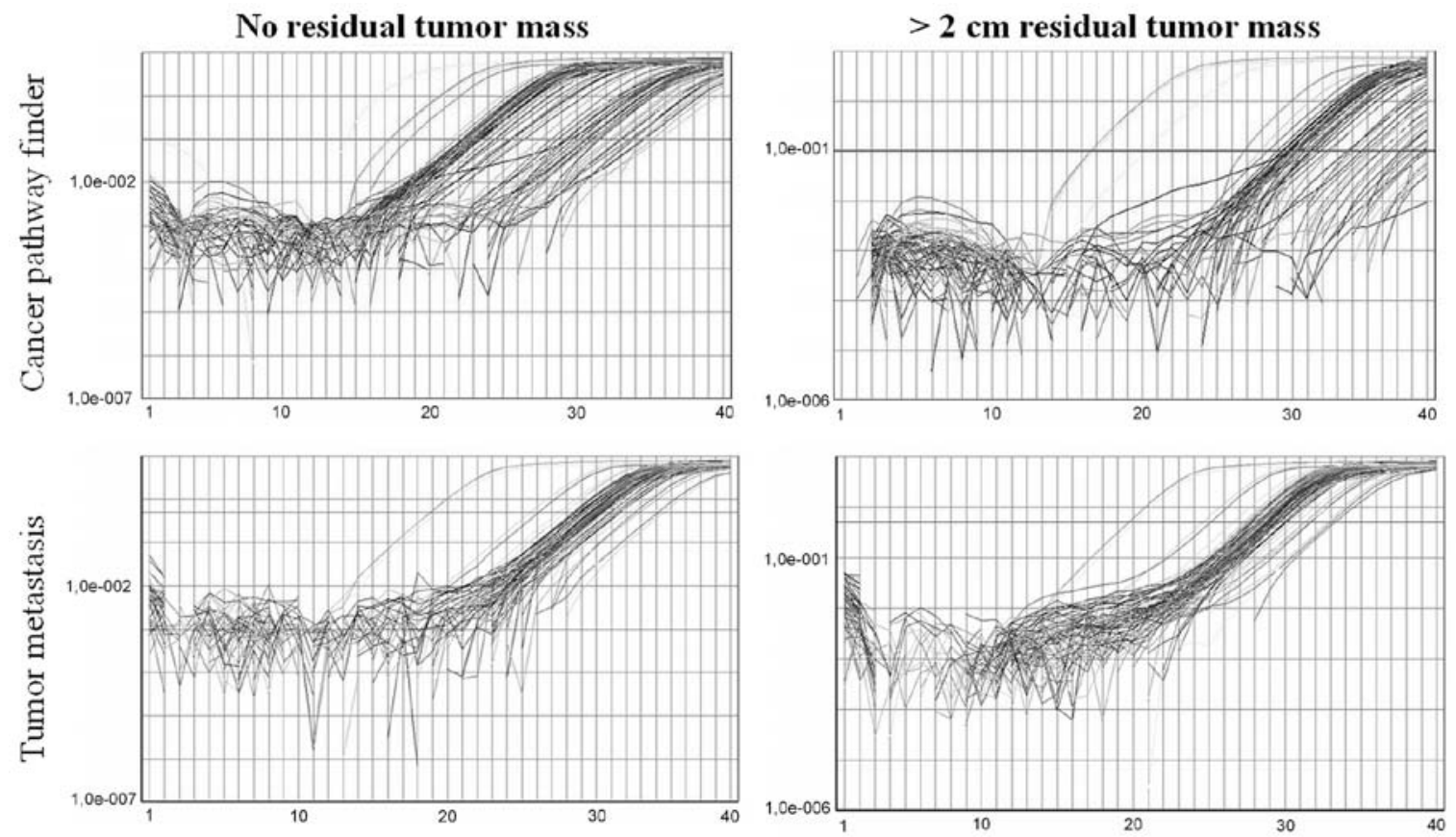

Figure 1. Real-time PCR plots from the Human Cancer PathwayFinder Array. Left column, representative examples from the control group (no residual tumor mass). Right column, typical examples from the test group ( $>2 \mathrm{~cm}$ residual tumor mass). Upper panel, results from the Human Cancer PathwayFinder Array. Lower panel, results from the Human Tumor Metastasis PCR array. As this figure shows there is a visual difference between separate subjects in the two groups.
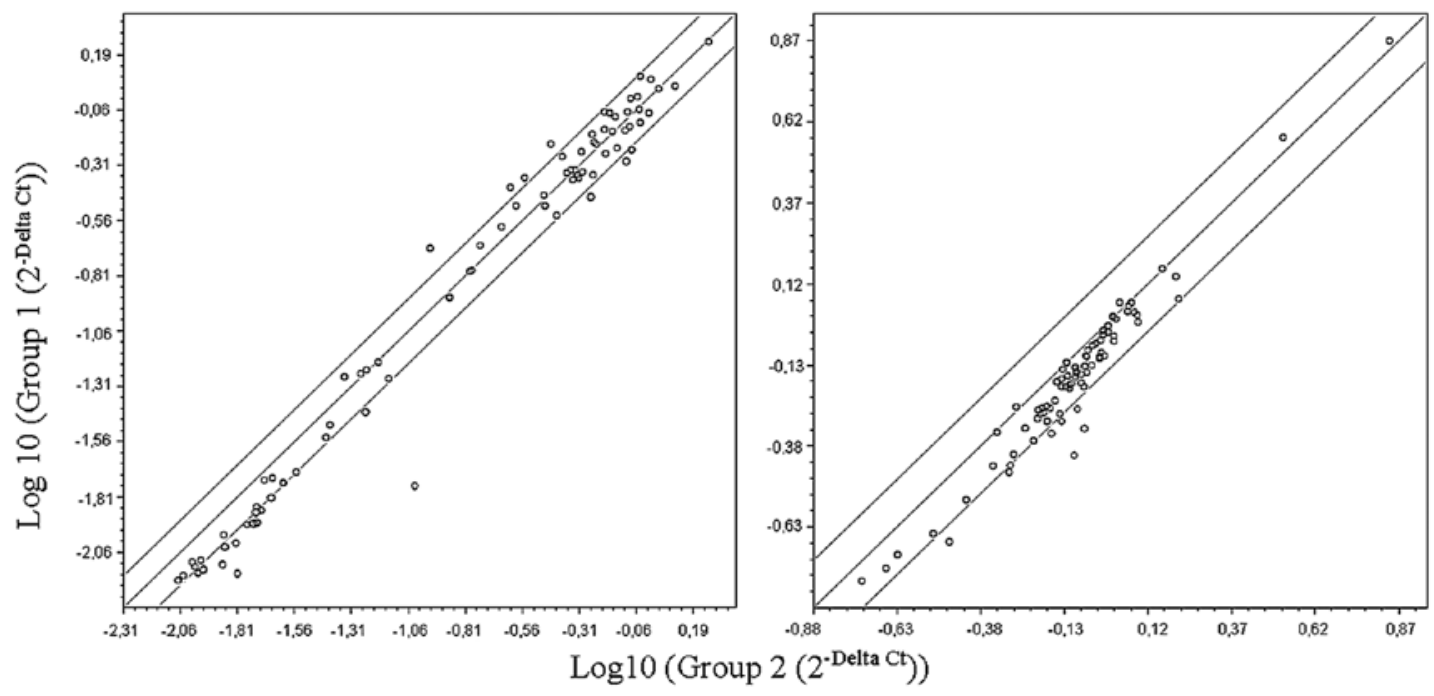

Figure 2. Relative expression of cancer related-genes in peripheral blood leukocytes from Group 1 (residual tumor present) and Group 2 (no residual tumor) Left panel, Human Cancer PathwayFinder PCRArray results. Right panel, Human Tumor Metastasis PCR Array results. The middle line represents no change in gene expression between the groups and the outer lines indicate a fold-change of \pm 1.40 . Group 1 are samples from the subjects with residual tumor mass; Group 2 consist of results from subjects that have had their tumor completely resected with surgery.

These calculations were carried out for all gene expressions in online software provided by the manufacturer (SABioscience, $\mathrm{RT}^{2}$ Profiler PCR array data analysis, 2008) (9). Fold upregulations or downregulations are calculated as: 1/fold-change.

\section{Results}

Basic characteristics of the study subjects are described in Table I, the two groups are fairly well-matched for age, but differ clinically according to the selection criteria. Nearly all the genes in both arrays were expressed similarly in both groups (Figs. 1 and 2). The relative mRNA expressions for the two PCR arrays are shown in Fig. 2. A majority of the differentially expressed genes in both arrays were downregulated (symbols under the lower line) and only a few were upregulated (symbols over the upper line). The six genes that showed significantly different expression between the two groups of patients are listed in Table II. In the Cancer PathwayFinder Array, MTA2 (metastasis associated 1 family, member 2) and TNF (tumor necrosis factor) were significantly downregulated 
Table II. Relative fold-change between the residual tumor group (Group 1) and the no residual tumor group (Group 2).

\begin{tabular}{|c|c|c|c|c|c|c|c|c|c|}
\hline \multirow[b]{2}{*}{ Gene } & \multirow[b]{2}{*}{ PCR array } & \multicolumn{2}{|c|}{ AVG $\Delta \mathrm{C}_{\mathrm{t}}$} & \multicolumn{2}{|c|}{$2^{-\Delta \mathrm{Ct}}$} & \multicolumn{2}{|c|}{$2^{-\Delta C t}$ fold-change } & \multirow[b]{2}{*}{ P-value } & \multirow{2}{*}{$\begin{array}{c}\text { Fold- } \\
\text { downregulated, } \\
\text { Group 1/Group } 2\end{array}$} \\
\hline & & Group 1 & Group 2 & Group 1 & Group 2 & Group 1/Group 2 & $95 \% \mathrm{CI}$ & & \\
\hline CTNNA1 & HTM & 0.15 & -0.06 & 0.90 & 1.04 & 0.87 & $(0.57,1.17)$ & 0.019 & -1.15 \\
\hline IL1B & HTM & 0.37 & 0.08 & 0.77 & 0.95 & 0.82 & $(0.35,1.29)$ & 0.042 & -1.22 \\
\hline KISS1 & HTM & 0.34 & 0.03 & 0.79 & 0.98 & 0.81 & $(0.40,1.22)$ & 0.019 & -1.24 \\
\hline MMP10 & HTM & -0.07 & -0.29 & 1.05 & 1.22 & 0.86 & $(0.48,1.24)$ & 0.048 & -1.16 \\
\hline MTA2 & $\mathrm{HCPF}$ & 0.80 & 0.25 & 0.57 & 0.84 & 0.68 & $(-0.08,1.44)$ & 0.039 & -1.46 \\
\hline TNF & $\mathrm{HCPF}$ & 1.50 & 0.86 & 0.35 & 0.55 & 0.64 & $(-0.24,1.52)$ & 0.046 & -1.57 \\
\hline
\end{tabular}

HTM, human tumor metastasis PCR array; HCPF, human cancer pathwayfinder PCR array). Changes in expression of cancer and metastasis related genes between Group 1 (residual tumor present) and Group 2 (no residual tumor). Only genes that showed significant differences in expression are tabulated here.

in the poor prognosis group. In the Tumor Metastasis Array, CTNNA1 ( $\alpha$-catenin), IL1B (Interleukin 1 $\beta$ ), KISS1 (KiSS-1 metastasis-suppressor) and MMP10 (matrix metalloproteinase 10/Stromelysin 2) were significantly downregulated. No genes in these two arrays were significantly upregulated in the poor prognosis group.

\section{Discussion}

We have evaluated the relative expression in white blood cells of genes in several different cancer associated pathways; cell cycle control and DNA damage repair, apoptosis and cell senescence, signal transduction molecules and transcription factors, angiogenesis, cell adhesion genes, extracellular matrix proteins, cell growth and proliferation genes, transcription factors and regulators, invasion and metastasis genes.

We found six genes to be significantly downregulated in Group 1 compared to Group 2. All genes act in different biological processes and all play a role in cancer metastasis. CTNNA1 (also called $\alpha$-catenin), a protein involved in cellcell adhesion, acts with cadherins on the cell surface and $\beta$-catenin to form a bond to actin filaments (10), dysfunction in this adhesion complex may contribute to a dissociation of cells and cause cancer cells to dissociate from primary tumor and form metastases $(11,12)$. The metastasis suppressor gene KISS1 suppresses metastases of melanoma and breast carcinomas without affecting their tumorigenicity (13). Loss of the original function of this gene may contribute to more aggressive tumor cells (14), and a study in mice showed that KISS1 is a metastasis suppressor in ovarian cancer tissue (13). Another metastasis associated gene, MTA2, was downregulated; it is indirectly involved in the regulation of the p53 gene, which has been associated with loss of growth inhibition (15). The metalloproteinase (MMP) family consists of a group of proteins that are involved in the degradation of extracellular matrix in the normal tissue (16), and in our patients we found MMP10 to be downregulated. This enzyme specifically degrades proteoglycans, laminin, fibronectin, collagen III and IV (17). Several studies claim that MMP10 gene expression in cancerous tissue may be of diagnostic use, and may be a relevant pathway in carcinogenesis (17). In addition we found two cytokines to be downregulated, IL1B and TNF. Both these cytokines are important mediators of inflammation and involved in a number of cell functions, e.g. cell proliferation, differentiation, and apoptosis $(18,19)$.

The most important methodological feature of the present study was that we could study mRNA in a clinical material of patients with ovarian cancer assaying the mRNA in native, unstimulated leukocytes. This is also evident in Fig. 2 which shows that the great majority of the genes were contained within a narrow range of relative gene expression changes of up to 1.4-fold between Group 1 and Group 2. The fact that the data shown in Fig. 2 are well centered along the regression line of identity, and only a few genes stand out statistically significantly, demonstrates the internal consistency of the experimental set-up. One cannot expect big across-the-board differences between the groups, because they shared main clinical features: all had the same diagnosis, type of histology, and were age- and gender-matched. The main clinical distinction was the fact that one group had residual tumor mass and the other group had no residual tumor mass after surgery (Table I). Thus, we can assume that specific features of the biology of the tumor in the subjects with a residual tumor burden (Group 1) are associated with the downregulation of the expression of the six genes even in peripheral blood leukocytes. In a study on colorectal cancer patients using an Affymetrix whole blood genome expression array, overexpression of only 20 genes was found in peripheral white blood cells (WBC) in comparison to normal subjects (20). A similar study on WBC gene expression in lung cancer patients (8) identified a set of eight genes (5 downregulated and 3 upregulated) that differentiated cancer patients from healthy controls. Both these studies fit in nicely with our result, supporting the concept that gene expression signatures in peripheral blood samples can differentiate between subjects with or without tumor burden or even between cancer and healthy patients $(8,20)$.

A limitation of our study is that the amplitudes of the downregulation of the six genes were numerically small, but in fact are comparable to the typical changes obtained in expression of crucial genes in studies of, e.g. ischemia and inflammation in various organs (21-23). Also, we do not know whether some of our patients had circulating tumor cells in 
their blood which could have affected the results (7). Such an effect would however potentially bias towards upregulation, and our main findings pertain to downregulation of certain genes.

We conclude that in unstimulated native blood leukocytes, a panel of genes involved in inflammation and metastasis are significantly downregulated in ovarian cancer patients with residual tumor mass after primary surgery. Such a signature of genes may prove to be a useful tool. Preoperatively it might serve the surgeon as a guide to the biology of the tumor and postoperatively in the optimization of adjuvant treatment of ovarian cancer patients.

\section{Acknowledgements}

The authors wish to acknowledge the Lions' Cancer Research Foundation, and the Research Committee of Örebro County Council for giving financial support. Thanks to Berit Bermark, Eva Magnusson, and Lisa Nygren for collecting the blood samples and to Elisabeth Peippo for turning all the forms into a file.

\section{References}

1. Pellicciotta I, Yang CPH, Goldberg GL and Shahabi S: Epothilone B enhances Class I HLA and HLA-A2 surface molecule expression in ovarian cancer cells. Gynecol Oncol 122: 625-631, 2011

2. Mury D, Woelber L, Jung S, et al: Prognostic and predictive relevance of CA-125 at primary surgery of ovarian cancer. J Cancer Res Clin Oncol 137: 1131-1137, 2011.

3. Eskander RN and Randall LM: Bevacizumab in the treatment of ovarian cancer. Biologics 5: 1-5, 2011.

4. Gardner GJ and Jewell EL: Current and future directions of clinical trials for ovarian cancer. Cancer Control 18: 44-51, 2011.

5. Skírnisdóttir I and Sorbe B: Prognostic impact of body mass index and effect of overweight and obesity on surgical and adjuvant treatment in early-stage epithelial ovarian cancer. Int J Gynecol Cancer 18: 345-351, 2008.

6. Resnick KE, Alder H, Hagan JP, Richardson DL, Croce CM and Cohn DE: The detection of differentially expressed microRNAs from the serum of ovarian cancer patients using a novel real-time PCR platform. Gynecol Oncol 112: 55-59, 2009.

7. Poveda A, Kaye SB, McCormack R, et al: Circulating tumor cells predict progression free survival and overall survival in patients with relapsed/recurrent advanced ovarian cancer. Gynecol Oncol 122: 567-572, 2011.
8. Rotunno M, Hu N, Su H, et al: A gene expression signature from peripheral whole blood for stage I lung adenocarcinoma. Cancer Prev Res (Phila) 4: 1599-1608, 2011.

9. Livak KJ and Schmittgen TD: Analysis of relative gene expression data using real-time quantitative PCR and the 2(-Delta Delta C(T)) Method. Methods 25: 402-408, 2001.

10. Pokutta S, Drees F, Yamada S, Nelson WJ and Weis WI: Biochemical and structural analysis of alpha-catenin in cell-cell contacts. Biochem Soc Trans 36: 141-147, 2008.

11. Shibata H, Takano H, Ito M, et al: Alpha-catenin is essential in intestinal adenoma formation. Proc Natl Acad Sci USA 104: 18199-18204, 2007.

12. Davidson B, Berner A, Nesland JM, et al: E-cadherin and alpha-, beta-, and gamma-catenin protein expression is up-regulated in ovarian carcinoma cells in serous effusions. J Pathol 192: 460-469, 2000

13. Jiang Y, Berk M, Singh LS, et al: KiSS1 suppresses metastasis in human ovarian cancer via inhibition of protein kinase $\mathrm{C}$ alpha. Clin Exp Metastasis 22: 369-376, 2005.

14. Navenot JM, Fujii N and Peiper SC: KiSS1 metastasis suppressor gene product induces suppression of tyrosine kinase receptor signaling to Akt, tumor necrosis factor family ligand expression, and apoptosis. Mol Pharmacol 75: 1074-1083, 2009.

15. Toh Y and Nicolson GL: The role of the MTA family and their encoded proteins in human cancers: molecular functions and clinical implications. Clin Exp Metastasis 26: 215-227, 2009.

16. Nagase $\mathrm{H}$ and Woessner JF Jr: Matrix metalloproteinases. J Biol Chem 274: 21491-21494, 1999.

17. Miyata Y, Iwata T, Maruta S, et al: Expression of matrix metalloproteinase-10 in renal cell carcinoma and its prognostic role. Eur Urol 52: 791-797, 2007.

18. Landvik NE, Hart K, Skaug V, Stangeland LB, Haugen A and Zienolddiny S: A specific interleukin-1B haplotype correlates with high levels of IL1B mRNA in the lung and increased risk of non-small cell lung cancer. Carcinogenesis 30: 1186-1192, 2009.

19. Lee JY, Jung HJ, Song IS, et al: Protective role of cytosolic 2-cys peroxiredoxin in the TNF-[alpha]-induced apoptotic death of human cancer cells. Free Radic Biol Med 47: 1162-1171, 2009.

20. Galamb O, Sipos F, Solymosi N, et al: Diagnostic mRNA expression patterns of inflamed, benign, and malignant colorectal biopsy specimen and their correlation with peripheral blood results. Cancer Epidemiol Biomarkers Prev 17: 2835-2845, 2008.

21. Atz M, Walsh D, Cartagena P, et al: Methodological considerations for gene expression profiling of human brain. J Neurosci Methods 163: 295-309, 2007.

22. Armugam A, Cher CD, Lim K, Koh DC, Howells DW and Jeyaseelan K: A secretory phospholipase A2-mediated neuroprotection and anti-apoptosis. BMC Neurosci 10: 120, 2009.

23. Block T, Isaksson HS, Acosta S, Bjorck M, Brodin D and Nilsson TK: Altered mRNA expression due to acute mesenteric ischaemia in a porcine model. Eur J Vasc Endovasc Surg 41: 281-287, 2011. 